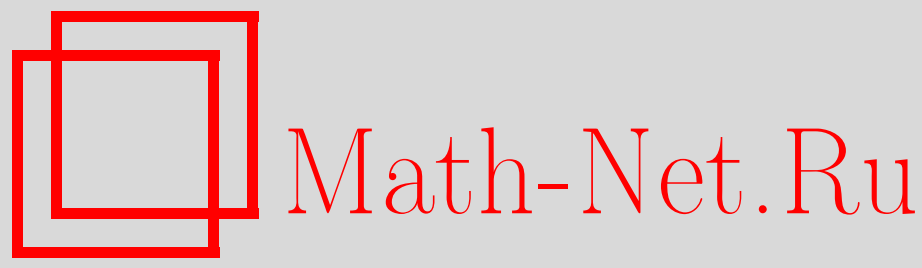

М. Гнатич, Ю. Хонконен, Т. Лучивянски, Влияние сжимаемости на процесс аннигиляции, ТМФ, 2013, том 176 , номер 1, 50-59

DOI: https://doi.org/10.4213/tmf8481

Использование Общероссийского математического портала Math-Net.Ru подразумевает, что вы прочитали и согласны с пользовательским соглашением http://www . mathnet.ru/rus/agreement

Параметры загрузки:

IP : 54.224 .135 .184

26 апреля 2023 г., 14:21:57

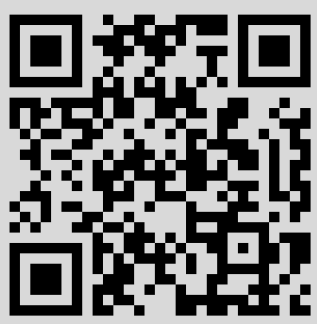


(C) 2013 г. $\quad$ М. Гнатич*†, Ю. Хонконен ${ }^{\ddagger}$, Т. Лучивянски ${ }^{* \dagger}$

\title{
ВЛИЯНИЕ СЖИМАЕМОСТИ НА ПРОЦЕСС АННИГИЛЯЦИИ
}

\begin{abstract}
С использованием ренормализационной группы в теории возмущений изучено влияние случайного поля скорости на кинетику реакции однотипной аннигиляции в случае критической размерности $d_{\mathrm{c}}=2$ и ниже ее. Переносимое поле скорости моделируется самоподобной в пространстве гауссовой переменной с конечным радиусом корреляции по времени (модель Антонова-Крейчнана). Учитывается эффект сжимаемости поля скоростей и анализируется модель вблизи ее критической размерности с помощью трехпараметрического разложения по $\epsilon, \Delta, \eta$. Здесь $\epsilon$ - отклонение от колмогоровского масштаба, $\Delta-$ отклонение от критической размерности пространства, $\eta$ - отклонение от параболического закона дисперсии. В зависимости от значений параметров разложения и параметра сжимаемости изучаемая модель может демонстрировать различные асимптотические (на больших временах) режимы, соответствующие инфракрасно-фиксированным точкам ренормализационной группы. Описываются возможные режимы и вычисляются скорости распада для среднего числа частиц в главном порядке теории возмущений.
\end{abstract}

Ключевые слова: процесс аннигиляции, модель Антонова-Крейчнана, пертурбативное ренормгрупповое приближение

DOI: $10.4213 / \operatorname{tmf} 8481$

\section{1. ВВЕДЕНИЕ}

В жидкой среде происходит целый ряд химических реакций, имеющих важное значение в различных химических, биологических или физических системах [1]-[3]. В этих процессах на реагирующие частицы воздействует не только диффузионное движение, но и внешнее течение жидкости. Обычный подход к таким реактивным течениям основан на некоторой комбинации уравнений реакции-транспорта.

* Institute of Experimental Physics of the Slovak Academy of Sciences, Košice, Slovakia. E-mail: hnatich@saske.sk

${ }^{\dagger}$ Faculty of Science, P. J. Šafárik University, Košice, Slovakia

${ }^{\ddagger}$ Department of Miltary Technology, National Defence University, Helsinki, Finland 
В настоящей работе мы сконцентрируемся на изучении реакции аннигиляции $A+A \rightarrow \varnothing$, которая является характерной моделью процессов реакции-диффузии. Для такого типа реакции в случае малых пространственных размерностей обычное описание с помощью кинетического уравнения скорости реакции является недостаточным, и необходимо учитывать влияние флуктуаций плотности [4]. Можно показать, что верхняя критическая размерность для этого процесса даже при отсутствии адвективного течения равна 2 благодаря флуктуациям плотности. Ренормгрупповой (РГ) подход успешно применялся для различных реактивных течений таких, как случайный дрейф, не зависящий от времени [5], поле скоростей, описываемое стохастическим уравнением Навье-Стокса [6], или ближний коррелированный потенциальный беспорядок [7]. Часто наблюдаются существенные отличия от эксперимента, их происхождение может быть вызвано наличием крупномасштабных анизотропий, эффектом сжимаемости или нарушением четности. Имеются важные различия [8], [9] между переносом скалярных величин типа плотности, с одной стороны, и индикаторов (температура, концентрация), с другой стороны, сжимаемым и несжимаемым течением. Ранее было показано, что сжимаемость может приводить к замедлению процессов переноса для скалярной примеси и к усилению перемежаемости. Эти эффекты можно понять как результат подавления разделения траекторий частиц, следовательно, мы ожидаем, что эффективно реагирующие частицы будут проводить значительно больше времени, находясь во взаимной близости, чем в случае несжимаемого течения. Таким образом, можно полагать, что скорость распада в этом случае будет больше, чем в случае несжимаемого течения. Отсюда следует, что было бы желательно рассмотреть эту качественную картину на количественном уровне. С этой целью мы используем модель Антонова-Крейчнана [10], [11] для описания переноса реагирующих частиц и представляем РГ-исследование вблизи ее критической размерности $d_{c}=2$. Все значимые физические величины рассчитаны в однопетлевом порядке, и в отличие от случая несжимаемого поля скоростей обнаружено, что уже в однопетлевом приближении флуктуации поля влияют на ренормировку константы скорости.

После краткого описания модели в разделе 2 результаты РГ-расчетов представлены в разделе 3 . В разделе 4 перечислены возможные крупномасштабные режимы; их физическая интерпретация дана в разделе 5 в качестве выводов.

\section{2. ТЕОРЕТИКО-ПОЛЕВАЯ МОДЕЛЬ}

Теоретико-полевое действие аннигиляционной реакции $A+A \stackrel{K_{0}}{\longrightarrow} \varnothing$ можно просто получить [12], применяя подход Доя [13]. Оно может быть записано в стандартном виде:

$$
\begin{aligned}
S_{1}= & -\int_{0}^{\infty} d t \int d \mathbf{x}\left\{\psi^{\dagger} \partial_{t} \psi-D_{0} \psi^{\dagger} \nabla^{2} \psi+\lambda_{0} D_{0}\left[2 \psi^{\dagger}+\left(\psi^{\dagger}\right)^{2}\right] \psi^{2}\right\}+ \\
& +n_{0} \int d \mathbf{x} \psi^{\dagger}(\mathbf{x}, 0)
\end{aligned}
$$

где $D_{0}$ - константа диффузии реагирующих частиц, и по соображениям размерности, мы написали произведение $\lambda_{0} D_{0}$ вместо константы скорости $K_{0}$. Последний 
член в действии соответствует начальным условиям, которые традиционно выбираются в виде распределения Пуассона.

В сжимаемом поле скорости имеются два типа задач диффузии-адвекции: перенос поля плотности и перенос поля индикатора [14]. Здесь мы анализируем случай переноса поля плотности [15]. В действии (1) это соответствует замене $\psi^{\dagger} \partial_{t} \psi \rightarrow$ $\psi^{\dagger}\left[\partial_{t} \psi+(\nabla \cdot \mathbf{v} \psi)\right]$, где $\mathbf{v}=\mathbf{v}(\mathbf{x}, t)$ - переносимое поле скорости. Согласно работе [11] предположим, что $\mathbf{v}$ - случайная гауссова переменная с нулевым средним и коррелятором (в частотно-импульсном представлении)

$$
\left\langle v_{0 i} v_{0 j}\right\rangle_{0}=\frac{g_{0} D_{0}^{3} k^{2-2 \Delta-2 \epsilon-2 \eta}}{\omega^{2}+\left(u_{0} D_{0} k^{2-\eta}\right)^{2}}\left[P_{i j}(\mathbf{k})+\alpha Q_{i j}(\mathbf{k})\right]
$$

где $g_{0}$ - константа связи, а показатели степени $\epsilon, \Delta$ и $\eta$ играют роль малых параметров разложения. Они могут рассматриваться как аналоги параметра разложения $\epsilon=4-d$, используемого в теории критических явлений. Однако здесь следует понимать $\epsilon$ как отклонение показателя степени от показателя в скейлинге Колмогорова [16], в то время как $\Delta$ определяется как отклонение от размерности пространства $d_{\mathrm{c}}=2$ с помощью соотношения $d=2+2 \Delta$, а показатель $\eta$ связан с обратной величиной времени корреляции для волнового числа $k$. Параметр $u_{0}$ служит для обозначения фиксированных точек, и его можно интерпретировать как отношение времени корреляции скорости к времени скалярного цикла [17]. В формуле (2), кроме стандартного (несжимаемого) поперечного проекционного оператора $P_{i j}(\mathbf{k})=\delta_{i j}-k_{i} k_{j} / k^{2}$, был введен продольный оператор $Q_{i j}(\mathbf{k})=k_{i} k_{j} / k^{2}$. Положительный параметр $\alpha$, необходимый для того, чтобы коррелятор $\langle v v\rangle$ был положительно определен, задает степень сжимаемости. Случай несжимаемого поля получается, если положить $\alpha=0$.

Модель Антонова-Крейчнана для адвективного поля v включает два особо интересных случая:

1) в пределе $u_{0} \rightarrow \infty, g_{0}^{\prime} \equiv g_{0} / u_{0}^{2}=$ const мы получаем "модель быстрых изменений" $D_{v}(\omega, \mathbf{k}) \rightarrow g_{0}^{\prime} D_{0} k^{-2-2 \Delta-2 \epsilon+\eta}$, которая характеризуется временной природой коррелятора скорости типа белго шума;

2) предел $u_{0} \rightarrow 0, g_{0}^{\prime \prime} \equiv g_{0} / u_{0}=$ const соответствует случаю замороженного поля скорости $D_{v}(\omega, \mathbf{k}) \rightarrow g_{0}^{\prime \prime} D_{0}^{2} \pi \delta(\omega) k^{2 \Delta-2 \epsilon}$, когда поле скорости вморожено (не зависит от времени).

Процедуру усреднения по полю скорости $\mathbf{v}(x)$ можно провести с помощью функционала действия

$$
S_{2}=-\frac{1}{2} \int d t d \mathbf{x} \int d t^{\prime} d \mathbf{x}^{\prime} \mathbf{v}(t, \mathbf{x}) D_{v}^{-1}\left(t-t^{\prime}, \mathbf{x}-\mathbf{x}^{\prime}\right) \mathbf{v}\left(t^{\prime}, \mathbf{x}^{\prime}\right)
$$

где $D_{v}^{-1}$ - обратный к коррелятору (2) оператор (в смысле образа Фурье). Математическое ожидание любой физической наблюдаемой величины можно вычислить с помощью полного весового функционала $\mathcal{W}\left(\psi^{\dagger}, \psi, \mathbf{v}\right)=e^{S_{1}+S_{2}}$, где $S_{1}$ и $S_{2}$ - функционалы действия (1) и (3). 
ТАБлицА 1. Канонические размерности для параметров и полей модели.

\begin{tabular}{|c|c|c|c|c|c|c|c|c|}
\hline$Q$ & $\psi$ & $\psi^{\dagger}$ & $v$ & $D_{0}$ & $u_{0}$ & $\lambda_{0}$ & $g_{0}$ & $\alpha, g, u, \lambda$ \\
\hline$d_{Q}^{k}$ & $d$ & 0 & -1 & -2 & $\eta$ & $-2 \Delta$ & $2 \epsilon+\eta$ & 0 \\
\hline$d_{Q}^{\omega}$ & 0 & 0 & 1 & 1 & 0 & 0 & 0 & 0 \\
\hline$d_{Q}$ & $d$ & 0 & 1 & 0 & $\eta$ & $-2 \Delta$ & $2 \epsilon+\eta$ & 0 \\
\hline
\end{tabular}

\section{3. УЛЬТРАФИОЛЕТОВАЯ РЕНОРМИРОВКА}

Включение продольной части $Q$ в коррелятор поля скорости не влияет на РГанализ, разработанный для такой модели [18]. Следовательно, мы просто упомянем основные шаги теоретического описания и отклонения от него, вызванные нарушением условия сжимаемости. Все канонические размерности полей и параметров приведены в табл. 1. Единственное отличие от случая несжимаемого поля заключается в том, что теперь поле скорости должно быть ренормировано [11]. Если следовать работам [6], [11], [18], то легко доказать, что рассматриваемая модель является мультипликативно ренормируемой и может быть сделана ультрафиолетовоконечной (УФ-конечной) с помощью следующей ренормировки:

$$
D_{0}=D Z_{D}, \quad g_{0}=g \mu^{2 \epsilon+\eta} Z_{g}, \quad u_{0}=u \mu^{\eta} Z_{u}, \quad \lambda_{0}=\lambda \mu^{-2 \Delta} Z_{D}^{-1} Z_{\lambda}, \quad \mathbf{v}_{0}=\mathbf{v} Z_{v}
$$

с дополнительными ограничениями

$$
Z_{g} Z_{D}^{3}=1, \quad Z_{u} Z_{D}=1, \quad Z_{g} Z_{D}^{3}=Z_{v}^{2},
$$

что является следствием отсутствия ренормировки нелокального члена (2). Нелокальный характер коррелятора скорости вызван нетривиальными корреляциями на масштабах импульса и частоты.

Полное ренормированное действие можно записать как

$$
\begin{aligned}
S_{\mathrm{R}}\left(\psi^{\dagger}, \psi, \mathbf{v}\right)=- & \int_{0}^{\infty} d t \int d \mathbf{x}\left\{\psi^{\dagger} \partial_{t} \psi-\psi^{\dagger} D Z_{D} \nabla^{2} \psi+\psi^{\dagger} Z_{v}(\boldsymbol{\nabla} \cdot \mathbf{v} \psi)\right]- \\
& \left.-Z_{\lambda} D \lambda\left[2 \psi^{\dagger}+\psi^{\dagger 2}\right] \psi^{2}\right\}- \\
& -\int d t d \mathbf{x} \int d t^{\prime} d \mathbf{x}^{\prime} \frac{v D_{v}^{-1} v}{2}+n_{0} \int d \mathbf{x} \psi^{\dagger}(\mathbf{x}, 0) .
\end{aligned}
$$

Расчет констант ренормировки в рамках теории возмущений в размерной регуляризации с применением схемы минимальных вычитаний осуществляется следующим образом [19]. Мы ограничиваемся первым порядком теории возмущений, и это приближение уже содержит первый нетривиальный эффект сжимаемости. Это можно увидеть из разложения (по теории возмущений) одночастично-неприводимой функции $\Gamma_{\psi^{\dagger} \psi^{2}}$, известной как вершина взаимодействия:

$$
\left.\Gamma_{\psi^{\dagger} \psi^{2}}\right|_{\omega=0, p^{2}=0}=-4 D \lambda Z_{\lambda} \mu^{-2 \Delta}+\frac{1}{2}
$$


где члены высшего порядка по константам связи опущены. Первая диаграмма в правой части уравнения (6) с физической точки зрения представляет процесс флуктуаций плотности, который отвечает за аннигиляцию двух частиц. Нетрудно видеть, что присутствие продольной части (случай сжимаемого поля скорости $\mathbf{v}$ ) пропагатора $\langle v v\rangle$ приводит к УФ-расходящемуся вкладу второй диаграммы в функцию (6), в то время как для моделей с несжимаемым полем скоростей $\mathbf{v}$ это приводит к УФ-сходящемуся вкладу. Физически этот вклад можно интерпретировать как процесс притяжения, который сближает частицы и направляет их в сток сжатия, что может привести к существенному возрастанию скорости реакции, как будет показано ниже. В схеме минимальных вычитаний константы ренормировки в однопетлевых вычислениях приобретают следующий вид:

$$
\begin{aligned}
& Z_{D}=1-\frac{g}{16 \pi u(1+u) \epsilon}\left[1+\alpha-\frac{2 \alpha}{1+u}\right] \\
& Z_{v}=1+\frac{\alpha g}{16 \pi u(1+u)^{2} \epsilon} \\
& Z_{\lambda}=1-\frac{\lambda}{4 \pi \Delta}-\frac{\alpha g}{16 \pi u(1+u) \epsilon}
\end{aligned}
$$

а константы $Z_{u}$ и $Z_{g}$ могут быть рассчитаны из соотношений (5). Предельный случай $\alpha=0$ находится в согласии с результатами (с однопетлевой точностью) для случая несжимаемого поля [18], а случай $g=0$ приводит к наличию флуктуаций только плотности [4].

Из соотношений (4) и (5) можно получить бета-функции $\beta_{g}, \beta_{u}$ и $\beta_{\lambda}$ с помощью стандартного определения $\beta_{g}=\left.\mu \partial_{\mu} g\right|_{0}$ (индекс 0 отвечает производным, взятым при фиксированных значениях затравочных (неренормированных) параметров):

$$
\beta_{g}=g\left[-2 \epsilon-\eta+3 \gamma_{D}-2 \gamma_{\mathbf{v}}\right], \quad \beta_{u}=u\left[-\eta+\gamma_{D}\right], \quad \beta_{\lambda}=\lambda\left[2 \Delta-\gamma_{\lambda}+\gamma_{D}\right],
$$

где аномальные размерности $\gamma_{F}$ величины $F$ определяются как [19]

$$
\gamma_{F}=\left.\mu \partial_{\mu} \ln Z_{F}\right|_{0}=\left(\beta_{g} \partial_{g}+\beta_{u} \partial_{u}+\beta_{\lambda} \partial_{\lambda}\right) \ln Z_{F}
$$

В работах [11], [17], [20] предполагалось, что вплоть до двухпетлевых расчетов непосредственное влияние параметра $\eta$ на аномальные размерности отсутствует. Отсюда следует, что можно использовать различные значения $\eta$ при расчетах феймановских диаграмм. В наших расчетах применялся самый простой выбор $\eta=0$.

Наконец, подставляя выражение (9) в определение (11), получаем аномальные размерности:

$$
\gamma_{D}=\frac{g}{8 \pi u(1+u)}\left[1+\alpha-\frac{2 \alpha}{u+1}\right], \quad \gamma_{v}=-\frac{\alpha g}{8 \pi u(1+u)^{2}}, \quad \gamma_{\lambda}=\frac{\alpha g}{8 \pi u(1+u)}-\frac{\lambda}{2 \pi} .
$$

\section{4. ИНФРАКРАСНО-УСТОЙЧИВЫЕ РЕЖИМЫ}

Нас интересуют инфракрасные (ИК) асимптотики небольших импульсов $\mathbf{p}$ и частот $\omega$ ренормированных функций или, что эквивалентно, больших относительных расстояний и временнь́х интервалов в $(t, \mathbf{x})$-представлении. Подобное поведение 
определяется ИК-устойчивой фиксированной точкой $g^{*}=\left(g_{1}^{*}, u^{*}, \lambda^{*}\right)$, значения которой определяются как нули бета-функций $(10): \beta\left(g^{*}\right)=0$. Считается, что фиксированная точка $g^{*}$ является ИК-устойчивой, если действительные части всех собственных значений матрицы $\Omega_{i j} \equiv \partial \beta_{i} /\left.\partial g_{j}\right|_{g=g^{*}}, i, j \in\{g, u, \lambda\}$, являются строго положительными.

Самый простой способ найти среднюю плотность числа частиц $n(t)=\langle\psi(t)\rangle$ заключается в том, чтобы вычислить ее из условия стационарности функционального преобразования Лежандра [21] производящего функционала, который получается с помощью замены неренормированного действия ренормированным в весовом функционале [6]. Это способ удобен тем, что можно избежать процедур суммирования, использующихся в работе [4] для того, чтобы учесть члены высшего порядка в $n_{0}$. В случае пространственно однородного решения это приводит к уравнению для средней плотности числа частиц вида

$$
n(t)=\frac{n_{0}}{1+2 \lambda u D t \mu^{-2 \Delta} n_{0}}
$$

с начальным условием $n(0)=n_{0}$, где $n_{0}-$ начальная плотность числа частиц. Так как поля $\psi$ и $\psi^{\dagger}$ неренормированы, уравнение Каллана-Симанзика для среднего числа частиц легко получается с помощью стандартной процедуры [6], [22]:

$$
\left[\left(2-\gamma_{D}\right) t \frac{\partial}{\partial t}+\sum_{g} \beta_{g} \frac{\partial}{\partial g}-d n_{0} \frac{\partial}{\partial n_{0}}+d\right] n\left(t, \mu, D, n_{0}, g\right)=0
$$

Если решать его с помощью метода характеристик, то можно показать (подробности будут опубликованы позднее [23]), что значение показателя распада $\beta$, который определяется с помощью асимптотического соотношения $n(t) \underset{t \rightarrow \infty}{\sim} t^{-\beta}$, дается выражением $\beta=1+\gamma_{\lambda}^{*} /\left(2-\gamma_{D}^{*}\right)$. Заметим, что в отличие от предыдущих исследований [6], [18] мы здесь имеем дело лишь с трехзарядовой $(\{g, u, \lambda\})$ теорией. Пассивный характер (нет обратного влияния на переносимое поле) реагирующей частицы проявляется в соотношениях $\partial_{\lambda} \beta_{g}=\partial_{\lambda} \beta_{u}=0$, вытекающих из формул (9) и (10). Эти соотношения очень сильно упрощают расчет собственных значений матрицы $\omega_{i j}$.

Анализ структуры с фиксированными точками показывает, что изучаемая система может демонстрировать один из десяти возможных ИК-режимов, перечисленных ниже. Во-первых, рассмотрим "модель быстрых изменений" $(u \rightarrow \infty)$. Если ввести удобные переменные $w=1 / u, g^{\prime}=g / u^{2}$, то можно записать соответствующие бета-функции в виде

$$
\beta_{g^{\prime}}=g^{\prime}\left[-2 \epsilon+\eta+\gamma_{D}-2 \gamma_{v}\right], \quad \beta_{w}=w\left[\eta-\gamma_{D}\right], \quad \beta_{\lambda}=\lambda\left[2 \Delta-\gamma_{\lambda}+\gamma_{D}\right],
$$

где аномальные размерности суть

$$
\gamma_{D}=\frac{g^{\prime}}{8 \pi(1+w)}\left[1+\alpha-\frac{2 \alpha w}{1+w}\right], \quad \gamma_{v}=-\frac{\alpha g^{\prime}}{8 \pi(1+w)^{2}}, \quad \gamma_{\lambda}=\frac{\alpha g^{\prime}}{8 \pi(1+w)}-\frac{\lambda}{2 \pi}
$$


"Модель быстрых изменений" соответствует фиксированной точке со значением $w^{*}=0$. В этом случае существуют четыре ИК-устойчивые фиксированные точки:

$$
\begin{aligned}
& \text { ФT-1 : } \quad g^{* *}=0, \quad \lambda^{*}=0, \\
& \Omega_{1}=\eta-2 \epsilon, \quad \Omega_{2}=\eta, \quad \Omega_{3}=2 \Delta, \\
& \beta=1 \text {; } \\
& \text { ФT-2 : } \quad g^{*}=0, \quad \lambda^{*}=-4 \pi \Delta, \\
& \Omega_{1}=\eta-2 \epsilon, \quad \Omega_{2}=\eta, \quad \Omega_{3}=-2 \Delta, \\
& \beta=1+\Delta \text {; } \\
& \text { ФТ-3 : } \quad g^{\prime *}=\frac{8 \pi(2 \epsilon-\eta)}{1+\alpha}, \quad \lambda^{*}=0, \\
& \Omega_{1}=2 \epsilon-\eta, \quad \Omega_{2}=2 \eta-2 \epsilon, \quad \Omega_{3}=2 \Delta+\frac{2 \epsilon-\eta}{1+\alpha}, \\
& \beta=\frac{2 \alpha+2-2 \epsilon+\eta}{(1+\alpha)(2-2 \epsilon+\eta)} \\
& \text { ФТ-4 : } \quad g^{\prime *}=\frac{8 \pi(2 \epsilon-\eta)}{1+\alpha}, \quad \lambda^{*}=2 \pi\left(2 \Delta+\frac{-2 \epsilon+\eta}{1+\alpha}\right) \text {, } \\
& \Omega_{1}=2 \epsilon-\eta, \quad \Omega_{2}=2 \eta-2 \epsilon, \quad \Omega_{3}=-2 \Delta-\frac{2 \epsilon-\eta}{1+\alpha}, \\
& \beta=\frac{2+2 \Delta}{2-2 \epsilon+\eta} \text {. }
\end{aligned}
$$

Чтобы проанализировать режим $u \rightarrow 0$ (вмороженное поле скорости), мы вводим новую переменную $g^{\prime \prime} \equiv g / u$. Тогда соответствующие бета-функции имеют вид

$$
\beta_{g^{\prime \prime}}=g^{\prime \prime}\left[-2 \epsilon+2 \gamma_{D}-2 \gamma_{v}\right], \quad \beta_{u}=u\left[-\eta+\gamma_{D}\right], \quad \beta_{\lambda}=\lambda\left[2 \Delta-\gamma_{\lambda}+\gamma_{D}\right],
$$

и аномальные размерности записываются как

$$
\gamma_{D}=\frac{g^{\prime \prime}}{8 \pi(1+u)}\left[1+\alpha-\frac{2 \alpha}{1+u}\right], \quad \gamma_{v}=-\frac{\alpha g^{\prime \prime}}{8 \pi(1+u)^{2}}, \quad \gamma_{\lambda}=\frac{\alpha g^{\prime \prime}}{8 \pi(1+u)}-\frac{\lambda}{2 \pi} .
$$

Такой вмороженный режим соответствует $u^{*}=0$, в этом случае также существуют четыре возможные ИК-устойчивые фиксированные точки:

$$
\begin{aligned}
& \text { ФT-5 : } \quad g^{\prime \prime *}=0, \quad \lambda^{*}=0 \text {, } \\
& \Omega_{1}=-2 \epsilon, \quad \Omega_{2}=-\eta, \quad \Omega_{3}=2 \Delta, \\
& \beta=1 \text {; } \\
& \text { ФT-6 : } \quad g^{\prime \prime *}=0, \quad \lambda^{*}=-4 \pi \Delta \text {, } \\
& \Omega_{1}=-2 \epsilon, \quad \Omega_{2}=-\eta, \quad \Omega_{3}=-2 \Delta, \\
& \beta=1+\Delta \\
& \text { ФT-7 : } \quad g^{\prime \prime *}=8 \pi \epsilon, \quad \lambda^{*}=0 \text {, } \\
& \Omega_{1}=2 \epsilon, \quad \Omega_{2}=-\eta+\epsilon(1-\alpha), \quad \Omega_{3}=2 \Delta+\epsilon(1-2 \alpha), \\
& \beta=\frac{2-(1-2 \alpha) \epsilon}{2-(1-\alpha) \epsilon} ;
\end{aligned}
$$




$$
\begin{array}{cl}
\text { ФT-8 : } \quad & g^{\prime \prime *}=8 \pi \epsilon, \quad \lambda^{*}=2 \pi[-2 \Delta+\epsilon(2 \alpha-1)], \\
& \Omega_{1}=2 \epsilon, \quad \Omega_{2}=-\eta+(1-\alpha) \epsilon, \quad \Omega_{3}=-2 \Delta+(2 \alpha-1) \epsilon, \\
& \beta=\frac{2+2 \Delta}{2-(1-\alpha) \epsilon} .
\end{array}
$$

Если не рассматривать особый выбор параметра $u$, имеет место нетривиальный случай. Будем считать, что $u$ конечен и отличен от нуля. Решая уравнение (10) для $u \neq 0, g \neq 0$, можно получить следующие значения для координат фиксированной точки:

$$
\frac{g^{*}}{8 \pi u^{*}\left(1+u^{*}\right)}=\frac{2 \epsilon-\eta}{1+\alpha}, \quad u^{*}=-1+\frac{\alpha(\eta-2 \epsilon)}{(1+\alpha)(\eta-\epsilon)} .
$$

Значение координаты $\lambda^{*}$ определяет два возможных режима. Фиксированная точка с нулевым значением $\lambda^{*}$ определяется как

$$
\text { ФТ-9 : } \quad \lambda^{*}=0, \quad \beta=\frac{2-\eta+\alpha(1+\epsilon-\eta)}{(1+\alpha)(2-\eta)}
$$

и является устойчивой в области

$$
(1-\alpha) \epsilon<\eta<\epsilon, \quad \Delta+\frac{(1+2 \alpha) \eta}{2(1+\alpha)}>\frac{\alpha \epsilon}{1+\alpha} .
$$

Фиксированная точка с ненулевым значением $\lambda^{*}$ есть

$$
\Phi \mathrm{T}-10: \quad \lambda^{*}=-4 \pi \Delta+\frac{2 \pi \alpha \epsilon}{1+\alpha}-2 \pi \eta \frac{1+2 \alpha}{1+\alpha}, \quad \beta=\frac{2+2 \Delta}{2-\eta},
$$

она является устойчивой в области

$$
(1-\alpha) \epsilon<\eta<\epsilon, \quad \Delta+\frac{(1+2 \alpha) \eta}{2(1+\alpha)}<\frac{\alpha \epsilon}{1+\alpha} .
$$

\section{5. ЗАКЛЮЧЕНИЕ}

Фиксированные точки ФТ-1 и ФТ-5 отвечают теории без взаимодействия (теории среднего поля и гауссовой теории), и, таким образом, предсказания этой теории должны согласовываться с предсказаниями, основанными на уравнении, рассмотренном в работе [4]. Из структуры фиксированных точек можно получить некоторые физические следствия. Во-первых, мы видим, что сжимаемость имеет непосредственное влияние на значение показателя распада (точки ФТ-3, ФТ-7-ФТ-9). Для некоторых режимов (ФТ-3, ФТ-7) это может привести к усилению процесса реакции (для обеих $\beta>1$ ) по сравнению с соответствующими режимами в случае несжимаемого поля [6], [18]. Как уже указывалось, этот факт можно объяснить наличием сжимаемых стоков, в которые затягиваются частицы (см. также работу [24]). Однако мы также наблюдаем, что, когда и флуктуации плотности, и сжимаемость являются существенными (для фиксированных точек ФТ-4, ФТ-10), плотность стремится подавить влияние сжимаемости.

"Реальная задача" соответствует выбору $\epsilon=\eta=4 / 3$, который приводит к знаменитому "закону пяти третьих" Колмогорова [16] для пространственной статистики 
скоростей. Нетрудно видеть, что этот режим может быть реализован с помощью фиксированных точек ФТ-9 или ФТ-10 в зависимости от значения параметра $\Delta$, либо, что эквивалентно, от размерности $d$ пространства. Из формул (13) и (14) видно, что имеется "критическое" значение $\Delta_{c}=-2 /(3+3 \alpha)$ для параметра $\Delta$, выше которого точка ФТ-9 является устойчивой, в то время как точка ФТ-10 является

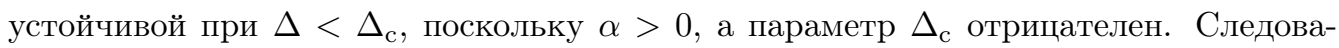
тельно, вблизи пространственной размерности $d_{\mathrm{c}}=2(\Delta=0)$, режим, представленный фиксированной точкой ФТ-9, должен быть реализован с показателем распада $\beta=(1+3 \alpha) /(1+\alpha)$. Таким образом, мы заключаем, что сжимаемость имеет сильное влияние на крупномасштабное асимптотическое поведение процесса аннигиляции и приводит к усилению его вблизи критической размерности $d_{\mathrm{c}}=2$. С другой стороны, флуктуации плотности опять ведут к подавлению сжимаемости (что можно видеть с помощью непосредственного изучения показателя $\beta$ для ФТ-10).

Благодарности. Работа поддержана Ministry of Education, Science, Research and Sport of the Slovak Republic (грант VEGA 1/0222/13), Centre of Excellency for Nanofluid, Institute of Experimental Physics, Slovak Academy of Sciences. Настоящая статья также создавалась в рамках программы "Cooperative phenomena and phase transition in nanosystems with perspective utilization in nano- and biotechnology" (проекты № 26220120033, 26110230061). Финансирование исследования и программы развития предоставил European Regional Development Fund.

\section{Список литературы}

[1] B. Derrida, V. Hakim, V. Pasquier, Phys. Rev. Lett., 75:4 (1995), 751-754, arXiv: hep-th/9505066.

[2] R. Kroon, H. Fleurent, R. Sprik, Phys. Rev. E, 47:4 (1993), 2462-2472.

[3] T. Tél, A. de Moura, C. Grebogi, G. Károlyi, Phys. Rep., 413:2-3 (2005), 91-196.

[4] B. P. Lee, J. Phys. A, 27:8 (1994), 2633-2652.

[5] M. W. Deem, J.-M. Park, Phys. Rev. E, 57:3 (1998), 2681-2685, arXiv: cond-mat/9707254; 58:3 (1998), 3223-3228, arXiv: cond-mat/9806195.

[6] M. Hnatich, J. Honkonen, Phys. Rev. E, 61 (2000), 3904-3911.

[7] M. J. E. Richardson, J. Cardy, J. Phys.A, 32:22 (1999), 4035-4045.

[8] A. Celani, A. Lanotte, A. Mazzino, Phys. Rev. E, 60:2 (1999), R1138-R1141.

[9] R. Benzi, M. H. Jensen, D. R. Nelson, P. Perlekar, S. Pigolotti, F. Toschi, Population dynamics in compressible flows, arXiv: 1203.6319.

[10] R. H. Kraichnan, Phys. Fluids, 11:5 (1968), 945-953.

[11] N. V. Antonov, Physica D, 144:3-4 (2000), 370-386.

[12] U. C. Täuber, M. Howard, B. P. Vollmayr-Lee, J. Phys. A, 38:17 (2005), R79-R131.

[13] M. Doi, J. Phys. A, 9:9 (1976), 1465-1478; J. Phys. A, 1976, 1479-1495.

[14] Л. Д. Ландау, Е. М. Лифшиц, Курс теоретической физики, т. 6: Гидродинамика, Наука, М., 2006.

[15] N. V. Antonov, J. Honkonen, Phys. Rev. E, 63:3 (2001), 036302, 7 pp., arXiv: nlin/0010029.

[16] У. Фриш, Турбулентность. Наследие А.Н. Колмогорова, ФАЗИС, М., 1998.

[17] L. Ts. Adzhemyan, N. V. Antonov, J. Honkonen, Phys. Rev. E, 66:3 (2002), 036313, arXiv: nlin/0204044.

[18] М. Гнатич, Ю. Хонконен, Т. Лучивянски, ТМФ, 169:1 (2011), 137-145. 
[19] J. Zinn-Justin, Quantum Field Theory and Critical Phenomena, International Series of Monographs on Physics, 77, Oxford Univ. Press, Oxford, 1989.

[20] N. V. Antonov, Phys. Rev. E, 60:6 (1999), 6691-6707.

[21] A. N. Vasil'ev, Functional Methods in Quum Field Theory and Statistical Physics, Gordon and Breach, Amsterdam, 1998.

[22] L. Ts. Adzhemyan, N.V. Antonov, A. N. Vasil'ev, The Field Theoretic Renormalization Group in Fully Developed Turbulence, Gordon and Breach, London, 1999.

[23] M. Hnatič, J. Honkonen, T. Lučivjanský, submitted to Eur. Phys. J. B.

[24] J. -P. Bouchaud, A. Georges, Phys. Rep., 195:4-5 (1990), 127-293. 